drawn using Kinovea and an average of seven consecutive steps was calculated for each angle. The four outcome measures were clustered using K-means cluster analysis $(n=2-10)$. Silhouette coefficients were used to detect optimal clustering. Results The cluster analysis led to the classification of two distinct subgroups (mean silhouette coefficient $=0.53$ ). Cluster 1 $(n=39)$ was characterized by higher foot inclination and tibia inclination at initial contact, higher knee flexion during midstance, and lower hip adduction during midstance compared to cluster $2(n=14)$. Fifteen out of $17(88 \%)$ shin injuries were classified in cluster 1 . Other injuries were more divided over both clusters. The ratio males/females was higher in cluster $1(44 \%)$ versus cluster $2(27 \%)$.

Conclusion This is the first study to classify subgroup profiles of running kinematics in recreational runners with an RRI based on two-dimensional video analysis. Two distinct subgroups were identified. This subclassification can help clinicians in their clinical reasoning process when evaluating kinematics of runners with an RRI and developing targeted gait-retraining strategies.

\section{TWO-DIMENSIONAL VIDEO ANALYSIS DURING RUNNING IN RECREATIONAL RUNNERS WITH AND WITHOUT RUNNING-RELATED KNEE INJURY}

\begin{abstract}
${ }^{1}$ Bart Dingenen*, ${ }^{2,3}$ Peter Malliaras, ${ }^{1}$ Tessa Janssen, ${ }^{1}$ Linde Ceyssens, ${ }^{1}$ Romy Vanelderen, ${ }^{3,4,5}$ Christian Barton. ${ }^{1}$ Rehabilitation Research Centre, Biomedical Research Institute, Faculty of Medicine and Life Sciences, UHasselt, Agoralaan A, Belgium; '2Department of Physiotherapy, School of Primary and Allied Health Care, Faculty of Medicine, Nursing and Health Science, Monash University, Australia; ${ }^{3}$ Complete Sports Care, Australia; ${ }^{5}$ Australia Department of Surgery, St Vincent's Hospital, University of Melbourne, Australia; ${ }^{4}$ La Trobe Sport and Exercise Medicine Research Centre, School of Allied Health, La Trobe University, Australia
\end{abstract}

\subsection{6/bjsports-2019-scandinavianabs.18}

Introduction The aim of this study was to compare running kinematics between recreational runners with and without running-related knee injury using two-dimensional video analysis. Materials and methods Forty-two recreational runners (18 injured, 24 non-injured) participated in the study. The injured group consisted of runners with anterior or lateral knee pain, resulting in altered running activity for at least one week. All participants ran on a treadmill at preferred speed. Digital videos were recorded in the frontal and sagittal plane with two iPads. Outcome measures included foot and tibia inclination at initial contact, and lateral trunk position, contralateral pelvic drop, femoral adduction, hip adduction, knee flexion and ankle dorsiflexion during midstance. All angles were manually drawn using Kinovea and an average of seven consecutive steps was calculated for each angle. Participant characteristics (gender, age, body weight, body length, body mass index, running volume before injury, running speed) and two-dimensional measured angles were compared between groups with independent t-tests (normally distributed) and the Mann Whitney $\mathrm{U}$ test (non-normally distributed).

Results There were no significant differences in participant characteristics between groups $(p>0.05)$. The injured group exhibited significantly more femoral adduction $(p=0.031)$ and hip adduction $(\mathrm{p}=0.004)$ during midstance, and significantly less foot inclination at initial contact $(p=0.026)$.

Conclusion Two-dimensional video analysis discriminated kinematics between runners with and without running-related knee injury. More femoral adduction and hip adduction during midstance, and less foot inclination at initial contact may provide gait retraining targets when treating runners with running-related knee injury.

\section{DO CLINICAL MEASURES RELATE TO RUNNING ASYMMETRIES IN PATIENTS WITH ACHILLES TENDINOPATHY?}

Patrick Corrigan*, Karin Grävare Silbernagel. University of Delaware, USA

10.1136/bjsports-2019-scandinavianabs.19

Introduction Achilles tendinopathy is an overuse injury that commonly sidelines runners and has a reinjury rate of $44 \%$. Aberrant loading patterns during the return-to-sports (RTS) phase may partially explain reinjury rates, but it remains unknown if clinical measures are associated with loading behaviors. Therefore, we aimed to establish relationships between side-to-side differences in running mechanics and side-to-side differences in clinical measures of pain, tendon structure, and calf muscle function.

Materials and methods 12 runners with Achilles tendinopathy were included (age: $44 \pm 11 \mathrm{y}$; height: $171 \pm 10 \mathrm{~cm}$; mass:70 $\pm 12 \mathrm{~kg}$; VISA-A score:71 \pm 10 ; current mileage: $40 \pm 29 \mathrm{~km} / \mathrm{wk}$ ). Participants completed a $7 \mathrm{~min}$ run at endurance pace $(2.9$ $\pm 0.3 \mathrm{~m} / \mathrm{s}$ ) on an instrumented treadmill with retroreflective markers affixed to their lower extremities. After a 6 min familiarization period, marker trajectories and ground reaction forces were sampled. Sagittal plane ankle joint moments and powers were calculated and a musculoskeletal model was used to estimate Achilles tendon loads. Pain-pressure threshold, tendon geometry, and calf muscle endurance were measured bilaterally with algometry, ultrasound imaging, and the heel-rise endurance test, respectively.

Results Side-to-side differences in pain-pressure threshold were significantly related to side-to-side differences in Achilles tendon loading rate $(\mathrm{r}=0.62 ; \mathrm{p}=0.03)$ and peak plantarflexion moment $(r=0.58, p=0.05)$. Side-to-side differences in peak eccentric ankle joint power were significantly related to sideto-side differences in tendon thickness $(r=0.59, \mathrm{p}=0.04)$ and cross-sectional area $(r=0.73 ; p=0.01)$. Side-to-side differences in calf muscle endurance was significantly related to Achilles tendon loading rate $(r=0.64 ; \mathrm{p}=0.03)$.

Conclusion Clinical measures of pain and calf muscle endurance relate to side-to-side differences in Achilles tendon loading rates during running, while tendon geometry relates to eccentric ankle joint power.

\section{THE ACHILLES TENDON TOTAL RUPTURE SCORE SHOULD BE USED WITH CAUTION THE FIRST 6 MONTHS AFTER INJURY}

\footnotetext{
${ }^{1,2}$ Maria Swennergren Hansen* ${ }^{*}{ }^{3}$ Katarina Nilsson Helander, ${ }^{3}$ Jón Karlsson, ${ }^{2}$ Kristoffe Weisskrichner Barfod. 'Physical Medicine and Rehabilitation Research-Copenhagen (PMRC); Department of Physical and Occupational Therapy, Copenhagen University Hospital Amager-Hvidovre, Denmark; ${ }^{2}$ Sports Orthopedic Research Center - Copenhagen (SORC-C), Department of Orthopedic Surgery, Copenhagen University Hospital Amager-Hvidovre, Denmark; ${ }^{3}$ Department of Orthopaedics, Sahlgrenska University Hospital, Sweden
}

\subsection{6/bjsports-2019-scandinavianabs.20}

Introduction The Achilles tendon Total Rupture Score (ATRS) is the most commonly used patient reported outcome in patients with an acute Achilles tendon rupture. The score 
contains ten items of which the last three concerns tasks that some patients cannot and some do not perform. No manual for the use of ATRS has been developed. The purpose was to investigate how ATRS responds at 4, 6 and 12 months after rupture and develop a manual for the use of ATRS.

Materials and methods This study was performed as a retrospective registry study analysing prospectively gathered data from the Danish Achilles tendon Database. The data was gathered 4, 6 and 12 months after rupture. The original score based on 10 items was compared with a score based on the first 7 items adjusted to the same scale as the original score. Density- and scatterplots were made and differences between the scores were tested by t-test or Mann-Whitney U test.

Results 2790 completed ATRS scores were included. The 7item score statistically significantly overestimated the value of the 10 items score at all time points $(\mathrm{p}<0.001)$ but only at 4 months the difference was clinical relevant (9.7points).

Conclusion The ATRS cannot be recommended for use at 4 months past rupture, as the last 3 items skew the score. If the ATRS is used before 6 months the last three items could be omitted. A manual for the use of the ATRS will be developed defining how and when the score should be applied.

\section{VALIDATION OF ACHILLES TENDON LENGTH MEASURE AND ACHILLES TENDON RESTING ANGLE IN RELATION TO COPENHAGEN ACHILLES LENGTH MEASURE}

\begin{abstract}
${ }^{1}$ Maria Swennergren Hansen* ${ }^{*}{ }^{1,2}$ Morten Tange Kristensen, ${ }^{3}$ Per Hölmich, ${ }^{3}$ Kristoffer Weisskrichner Barfod. 'Physical Medicine and Rehabilitation ResearchCopenhagen (PMR-C); Department of Physical and Occupational Therapy, Copenhagen University Hospital Amager-Hvidovre, Denmark; '2Department of Orthopaedic Surgery, Copenhagen University Hospital Amager-Hvidovre, Denmark; ${ }^{3}$ Sports Orthopedic Research Center - Copenhagen (SORC-C), Department of Orthopedic Surgery, Copenhagen University Hospital Amager-Hvidovre, Denmark
\end{abstract}

\subsection{6/bjsports-2019-scandinavianabs. 21}

Introduction Elongation of the Achilles tendon after rupture is a frequent and overlooked complication. The Achilles Tendon Length Measure (ATLM) and the Achilles Tendon Resting Angle (ATRA) are indirect length measures using the resting angle of the ankle. Copenhagen Achilles Length Measure (CALM) is a direct ultrasound measure. Examination of the association of elongation to valid clinical measures of the length of the Achilles tendon are needed. The purpose was to examine the concurrent validity of ATLM and ATRA in relation to CALM within one year of rupture.

Materials and methods The study was performed as a validity study. Data were collected from patients included in a randomized controlled trial. Mixed linear regression, controlling for time after injury, age and gender, was performed investigating the three models (dependent-independent): CALM-ATRA, CALM-ATLM and ATRA-ATLM.

Results 130 patients were included (23 women, 107 men) mean age 41.8 years (SD 10.5). All three regression models demonstrated a statistical significant $(\mathrm{p}<0.01)$ linear relationship. For each degree ATRA increased, CALM increased with $0.39 \mathrm{~mm}$. For each $\mathrm{cm}$ ATLM increased, CALM increased with $1.7 \mathrm{~mm}$. For each $\mathrm{cm}$ ATLM increase, ATRA increase with 1,6 degrees.

Conclusion ATRA and ATLM were found to have a linear relationship to CALM and seems valid as surrogate measurements for the assessment of tendon elongation after an Achilles tendon rupture.

\section{RUNNING A MARATHON - THE EFFECT ON ACHILLES TENDON STRUCTURE}

'Lucas Maciel Rabello*, ${ }^{1,2}$ Mathijs van Ark, ${ }^{1}$ Sophie Albers, ${ }^{3}$ Ron L Diercks, ${ }^{1} J o h a n n e s$ Zwerver, ${ }^{1,3}$ Inge van den Akker-Scheek. 'University of Groningen, University Medical Center Groningen, Department of Sports and Exercise Medicine, Netherlands; ${ }^{2}$ Hanze University of Applied Sciences Groningen, Department of Physiotherapy, Netherlands; ${ }^{3}$ University of Groningen, University Medical Center Groningen, Department of Orthopedics, Netherlands

\subsection{6/bjsports-2019-scandinavianabs.22}

Introduction More than half a million athletes were enrolled in a marathon run during the last years. During running the Achilles tendon is submitted to high loads, and multiple tensile strain cycles. There are no studies investigating the effect of a marathon in the structure of the Achilles tendon. Thus, the aim of this study was to investigate the short-term response of the Achilles tendon structure after running a marathon.

Materials and methods Ten male non-elite runners who participated in a marathon were included. Tendon structure was assessed before, 2 days and 7 days after a marathon using the ultrasound tissue characterization (UTC), an imaging tool which quantifies tendon organization dividing the structure into four different echo types (I-IV).

Results Two days after a marathon, no significant changes in tendon structure were observed. However, after 7 days both insertional and midportion structure changed significantly. At the insertion of the tendon there was a significant decrease in the percentage of echo types I, III and IV and a significant increase in the percentage of echo type II. The midportion of the tendon showed a significant decrease in the percentage of echo types III and IV and a significant increase in the percentage of echo type II.

Conclusion We observed that the effects of running a marathon on the Achilles tendon structure occured 7 days after the event. In a population of runners without tendon injuries, there seems to be a positive adaptation of the tendon to the load after running a marathon.

\section{EVALUATION OF IN-EAR SENSOR SYSTEMS FOR QUANTIFYING HEAD IMPACT EXPOSURE IN YOUTH FOOTBALL}

${ }^{1}$ Stian Bahr Sandmo*, 2,3,4 Andrew S Mclntosh, ${ }^{1}$ Thor Einar Andersen, ${ }^{5,6}$ Inga K Koerte, ${ }^{1}$ Roald Bahr. 'Department of Sports Medicine, Oslo Sports Trauma Research Center, Norwegian School of Sport Sciences, Norway; ${ }^{2}$ Federation University Australia, Australia; ${ }^{3}$ Monash University Accident Research Centre, Monash University, Australia; ${ }^{4}$ McIntosh Consultancy and Research, Australia; ${ }^{5}$ Department of Child and Adolescent Psychiatry, Psychosomatic and Psychotherapy, Ludwig-Maximilian University, Germany; ${ }^{6}$ Department of Psychiatry, Pscyhiatry Neuroimaging Laboratory, Brigham and Women's Hospital, Harvard Medical School, USA

\subsection{6/bjsports-2019-scandinavianabs.23}

Introduction Wearable sensor systems may be useful for measuring head-impact exposure. Here, we tested the validity of in-ear sensors developed to improve head coupling.

Methods First, the sensor was mounted to a Hybrid III headform (HIII) and impacted with a linear impactor or football. Peak linear acceleration (PLA), peak rotational acceleration (PRA) and peak rotational velocity (PRV) were obtained from both systems; random and systematic error were calculated using HIII as reference. Then, six youth football players wore sensors and performed a structured training protocol 